07

\title{
Дефекты с глубокими уровнями в фотоэлектрическом преобразователе с антиотражающей пленкой пористого кремния, сформированной окрашивающим химическим травлением
}

\author{
(C) В.В. Трегулов ${ }^{1}$, В.Г. Литвинов ${ }^{2}$, А.В. Ермачихин ${ }^{2}$ \\ ${ }^{1}$ Рязанский государственный университет им. С.А. Есенина, Рязань, Россия \\ ${ }^{2}$ Рязанский государственный радиотехнический университет, Рязань, Россия \\ E-mail: trww@yandex.ru
}

Поступило в Редакцию 16 ноября 2018г.

В окончательной редакции 16 ноября 2018 г.

Принято к публикации 21 ноября 2018г.

Методом токовой релаксационной спектроскопии глубоких уровней проведено исследование дефектов в полупроводниковой структуре фотоэлектрического преобразователя с $p-n$-переходом и антиотражающей пленкой пористого кремния, изготовленной с применением химического окрашивающего травления. Предложено объяснение влияния режимов формирования пленки пористого кремния на характер трансформации дефектов с глубокими уровнями и основные характеристики фотоэлектрического преобразователя.

DOI: 10.21883/PJTF.2019.04.47332.17597

Антиотражающая пленка пористого кремния (por-Si) на фронтальной поверхности кремниевого фотоэлектрического преобразователя (ФЭП) солнечной энергии с $p-n$-переходом позволяет существенно повысить его эффективность [1]. Наиболее популярным способом формирования антиотражающей пленки por-Si является анодное электрохимическое травление кремния в электролите на основе HF. Главный недостаток данного способа - малая длительность процесса травления (не более 20-25s при плотности тока не более $30 \mathrm{~mA} / \mathrm{cm}^{2}$ [1]). Необходимость точного контроля столь короткого промежутка времени может усложнить организацию технологического процесса изготовления ФЭП в условиях промышленного производства и привести к значительному разбросу основных характеристик выпускаемых изделий. Снижение скорости роста пленки por-Si может обеспечить метод химического окрашивающего травления, главная особенность которого заключается в отсутствии внешнего источника тока и применении травителя, содержащего наряду с HF раствор сильного окислителя [2]. При изготовлении ФЭП данный метод используется значительно реже, чем анодное электрохимическое травление [1]. В связи с этим особенности электрофизических процессов с учетом влияния дефектов с глубокими уровнями (ГУ) в таких ФЭП исследованы недостаточно.

Экспериментальные образцы изготавливались на кремниевых монокристаллических пластинах $p$-типа проводимости с удельным сопротивлением $1 \Omega \cdot \mathrm{cm}$ и ориентацией поверхности (100). Глубина залегания $n^{+}-p$-перехода составляла $0.5 \mu \mathrm{m}$. Пленка por-Si формировалась на поверхности $n^{+}$-слоя методом химического окрашивающего травления с использованием травителя $\mathrm{HF}: \mathrm{KMnO}_{4}: \mathrm{C}_{2} \mathrm{H}_{5} \mathrm{OH}$ (соотношение $1: 0.5: 1$ ). Окислитель $\mathrm{KMnO}_{4}$ вводился в состав травителя в виде водного раствора. Пленка por-Si формировалась при различных значениях концентрации $C$ водного раствора окислителя и разных длительностях процесса травления $t_{e t}$. Величины $C$ и $t_{e t}$ для исследованных образцов указаны в таблице. Контакты к пленке por-Si и кремниевой подложке $p$-типа формировались на противоположных поверхностях полупроводниковой структуры с помощью серебряной пасты ФС-1127.

Для исследования дефектов с ГУ применялся метод токовой релаксационной спектроскопии глубоких уровней (CDLTS) [3]. Заполнение и опустошение ГУ проводилось импульсным напряжением. Амплитуда напряжения импульса заполнения составляла $0 \mathrm{~V}$, опустошение ГУ осуществлялось при напряжении обратного смещения 2 V. При выбранном режиме заполнения ГУ происходит преимущественно заполнение ловушек основных носителей заряда - дырок. Режим измерения был выбран из соображений оптимального соотношения сигнал/шум. Сигнал релаксации тока анализировался с помощью весовой функции „lock-in“.

Спектры CDLTS исследуемых образцов, измеренные при постоянной времени $1.18 \mathrm{~ms}$, показаны на рис. $1-3$, для сравнения также представлен спектр ФЭП c $n^{+}-p$-переходом без пленки por-Si (образец № 1). Основные параметры ГУ (энергия активации $E_{t}$ с указанием погрешности и концентрация $N_{t}$ ) приведены в таблице. Достаточно большой разброс величин $E_{t}$ может быть обусловлен наложением сигналов от нескольких ГУ с близкими значениями $E_{t}$.

Спектры CDLTS образцов, исследованных в настоящей работе, и образцов с пленкой por-Si, сформированной анодным электрохимическим травлением [4], имеют некоторые сходства. Общими для обоих типов образцов являются ГУ $\mathrm{H3}$ и $\mathrm{H5}$. 


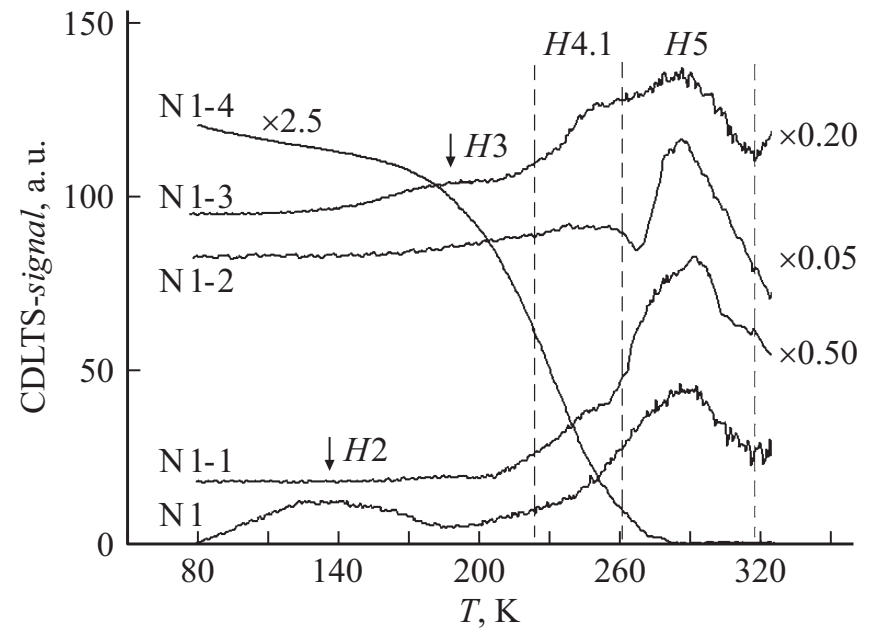

Pис. 1. Спектры CDLTS образцов № 1-1, 1-2, 1-3, 1-4, изготовленных при $C=0.025 \mathrm{M}$, а также образца № 1 без пленки por-Si.

На спектрах всех образцов присутствует ГУ $H 5$ (см. таблицу). Травление при $C=0.025 \mathrm{M}$ и величинах $t_{e t}=0.5$ и $1.0 \mathrm{~min}$ приводит к существенному снижению $N_{t}$ ГУ $H 5$ по сравнению с аналогичной величиной для образца ФЭП без пленки por-Si (образец № 1) (рис. 1). Также на CDLTS-спектрах образцов, в которых травление проводилось при $C=0.025 \mathrm{M}$, присутствует ГУ H4.1. В образцах с пленкой por-Si, изготовленной с применением анодного электрохимического травления [4], ГУ $H 4.1$ не наблюдался. Травление при $C=0.025 \mathrm{M}$ и $t_{e t}=0.5 \mathrm{~min}$ не приводит к существенному изменению $N_{t}$ ГУ $H 4.1$ по сравнению с величиной $N_{t}$ для образца № 1. С увеличением $t_{e t}$ до $1.0 \min N_{t}$ ГУ $H 4.1$ заметно снижается. Отмеченное снижение $N_{t}$ ГУ $H 5$ и $H 4.1$ может объясняться влиянием процесса травления. Травление полупроводникового материала более интенсивно протекает по дефектным областям, что может приводить к снижению $N_{t}$ [5]. Снижение $N_{t}$ также может быть связано с эффектом пассивации дефектов с ГУ при формировании пленки por-Si [6-8]. Этим также может объясняться отсутствие ГУ $H 2$ на спектрах образцов после травления (в отличие от образца № 1). Увеличение времени $t_{e t}$ до $2.0 \mathrm{~min}$ при $C=0.025 \mathrm{M}$ (образец № 13) приводит к появлению ГУ Н3, концентрация ГУ $H 4.1$ увеличивается на порядок величины, а концентрация ГУ $H 5$ незначительно возрастает по сравнению с таковыми для образца № 1-2. Это можно объяснить появлением локальных проколов плоскости $n^{+}-p$-перехода в результате травления. Дальнейшее увеличение $t_{e t}$ до $3.0 \mathrm{~min}$ при $C=0.025$ М (образец № 1-4) приводит к существенной трансформации спектра CDLTS. Форма спектра для образца № 1-4 характерна для поверхностных состояний с концентрацией, равной или превышающей концентрацию мелкой акцепторной примеси в базовой области $n^{+}-p$-перехода. Это может свидетельствовать о существенных нарушениях плоскости $n^{+}-p$-перехода в результате травления. Аналогичная ситуация характерна для образцов № 2-3 (рис. 2) и № 3-3 (рис. 3), изготовленных при $t_{e t}=3.0 \mathrm{~min}$ и значениях $C=0.040$ и $0.050 \mathrm{M}$ соответственно.

Ha CDLTS-спектрах образцов № 2-1 и 2-2, изготовленных при $C=0.040 \mathrm{M}$, присутствует только один ГУ $H 5$ (рис. 2), причем образец № 2-2, изготовленный при $t_{e t}=1.0 \mathrm{~min}$, характеризуется наиболее низкой концентрацией ГУ $H 5$ из всех исследованных в настоящей работе образцов.

На CDLTS-спектре образца № 3-1 с пленкой por-Si, сформированной при $C=0.050 \mathrm{M}$ и $t_{e t}=0.5 \mathrm{~min}$, присутствует единственный ГУ $-H 5$ (рис. 3). Травление в указанном режиме позволяет существенно снизить $N_{t}$ ГУ Н5 для образца № 3-1 по сравнению с таковой для образца № 2-1, изготовленного при том же значении $t_{e t}$ и $C=0.040 \mathrm{M}$. Увеличение $t_{e t}$ до $1.0 \mathrm{~min}$ при $C=0.050 \mathrm{M}$ (образец № 3-2) приводит к росту $N_{t}$ ГУ $H 5$ более чем на порядок величины. Также появляется широкий пик

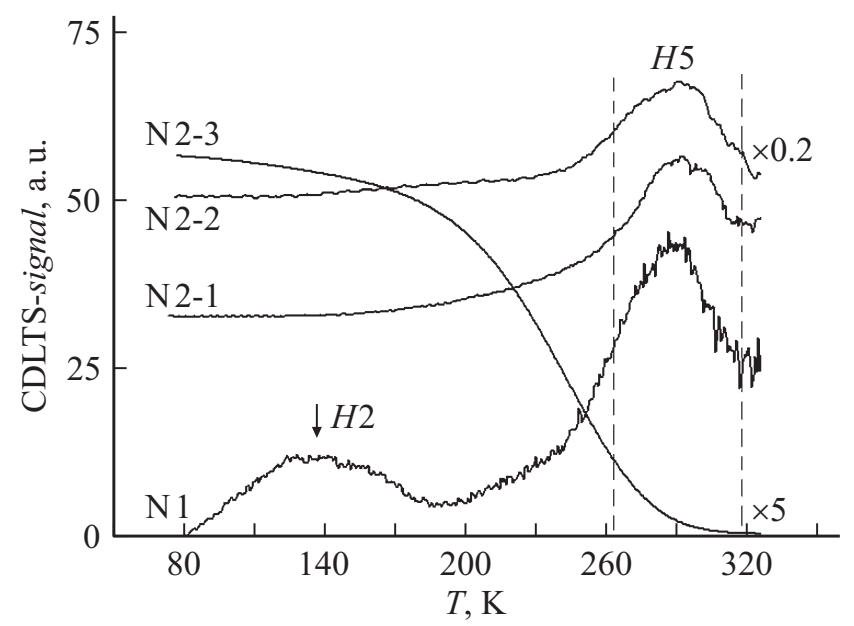

Рис. 2. Спектры CDLTS образцов № 2-1, 2-2, 2-3, изготовленных при $C=0.040 \mathrm{M}$, а также образца № 1 без пленки por-Si.

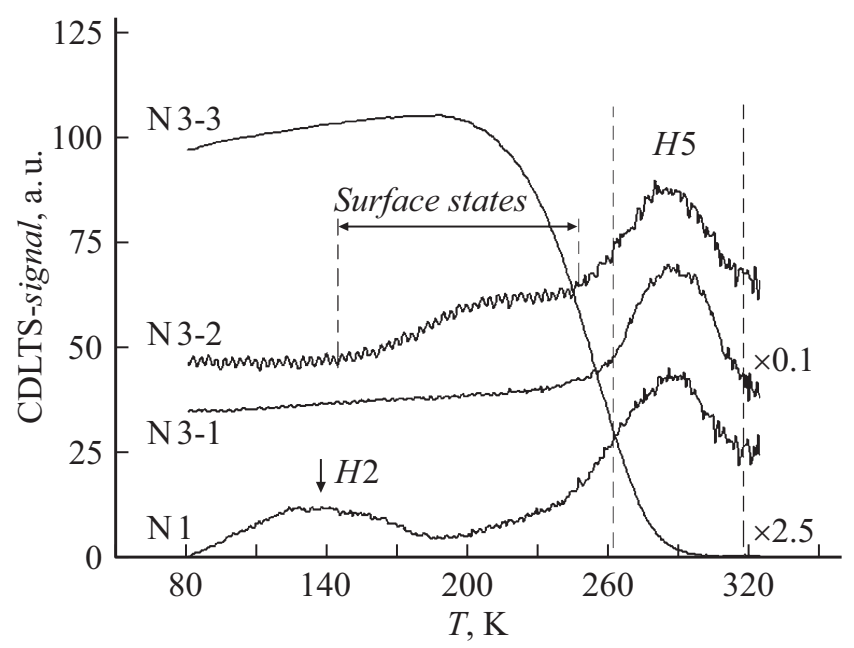

Рис. 3. Спектры CDLTS образцов № 3-1, 3-2, 3-3, изготовленных при $C=0.050 \mathrm{M}$, а также образца № 1 без пленки por-Si. 
Сведения о режимах формирования пленки por-Si экспериментальных образцов, параметрах ГУ и характеристиках ФЭП

\begin{tabular}{|c|c|c|c|c|c|c|c|c|c|}
\hline \multirow{2}{*}{$\begin{array}{l}\text { Номер } \\
\text { образца }\end{array}$} & \multirow{2}{*}{$C, \mathrm{M}$} & \multirow{2}{*}{$\begin{array}{l}t_{e t}, \\
\min \end{array}$} & \multicolumn{3}{|c|}{ Параметры ГУ } & \multicolumn{4}{|c|}{ Характеристики ФЭП } \\
\hline & & & ГУ & $\begin{array}{l}E_{t} \\
\mathrm{eV}\end{array}$ & $\begin{array}{c}N_{t} \\
\mathrm{~cm}^{-3}\end{array}$ & $\begin{array}{l}U_{o c} \\
\mathrm{mV}\end{array}$ & $\begin{array}{c}J_{s c}, \\
\mathrm{~mA} / \mathrm{cm}^{2}\end{array}$ & $\begin{array}{l}F F, \\
\text { a.u. }\end{array}$ & $\begin{array}{l}\eta \\
\%\end{array}$ \\
\hline 1 & - & - & $\begin{array}{c}H 2 \\
H 4.1 \\
H 5\end{array}$ & $\begin{array}{l}0.18 \pm 0.05 \\
0.37 \pm 0.05 \\
0.49 \pm 0.06\end{array}$ & $\begin{array}{l}2.78 \cdot 10^{15} \\
3.26 \cdot 10^{15} \\
8.16 \cdot 10^{15}\end{array}$ & 505 & 13.64 & 0.77 & 7.08 \\
\hline $1-1$ & 0.025 & 0.5 & $\begin{array}{c}H 4.1 \\
H 5\end{array}$ & $\begin{array}{l}0.37 \pm 0.05 \\
0.49 \pm 0.06\end{array}$ & $\begin{array}{l}3.35 \cdot 10^{15} \\
7.05 \cdot 10^{15}\end{array}$ & 510 & 30.19 & 0.78 & 12.44 \\
\hline $1-2$ & 0.025 & 1.0 & $\begin{array}{c}H 4.1 \\
H 5\end{array}$ & $\begin{array}{l}0.37 \pm 0.05 \\
0.49 \pm 0.06\end{array}$ & $\begin{array}{l}1.27 \cdot 10^{14} \\
5.31 \cdot 10^{14}\end{array}$ & 520 & 33.39 & 0.79 & 13.72 \\
\hline $1-3$ & 0.025 & 2.0 & $\begin{array}{c}H 3 \\
H 4.1 \\
H 5\end{array}$ & $\begin{array}{l}0.27 \pm 0.04 \\
0.37 \pm 0.05 \\
0.49 \pm 0.06\end{array}$ & $\begin{array}{l}5.77 \cdot 10^{14} \\
1.42 \cdot 10^{15} \\
5.91 \cdot 10^{14}\end{array}$ & 520 & 32.11 & 0.78 & 13.42 \\
\hline $1-4$ & 0.025 & 3.0 & & зерхностные & яния & 509 & 12.42 & 0.66 & 4.90 \\
\hline $2-1$ & 0.040 & 0.5 & $H 5$ & $0.49 \pm 0.06$ & $4.05 \cdot 10^{15}$ & 511 & 31.43 & 0.78 & 13.13 \\
\hline $2-2$ & 0.040 & 1.0 & $H 5$ & $0.49 \pm 0.06$ & $2.8 \cdot 10^{14}$ & 521 & 34.58 & 0.78 & 14.10 \\
\hline $2-3$ & 0.040 & 3.0 & & зерхностные & яния & 483 & 7.12 & 0.64 & 2.62 \\
\hline $3-1$ & 0.050 & 0.5 & $H 5$ & $0.49 \pm 0.06$ & $7.95 \cdot 10^{14}$ & 514 & 34.67 & 0.77 & 13.40 \\
\hline $3-2$ & 0.050 & 1.0 & H5 & $\begin{array}{r}\text { зерхностные } \\
0.49 \pm 0.06\end{array}$ & $\begin{array}{l}\text { яния } \\
8.15 \cdot 10^{15}\end{array}$ & 492 & 24.45 & 0.77 & 10.04 \\
\hline $3-3$ & 0.050 & 3.0 & & зерхностные & яния & 467 & 4.85 & 0.51 & 1.80 \\
\hline
\end{tabular}

в области энергий активации ГУ $H 3$ и $H 4.1$, который может быть связан с возникновением поверхностных состояний с плотностью $\sim 2.0 \cdot 10^{11} \mathrm{~cm}^{-2}$ в плоскости $n^{+}-p$-перехода (рис. 3). Дальнейшее увеличение $t_{e t}$ до $3.0 \mathrm{~min}$ при $C=0.050 \mathrm{M}$ (образец № 3-3) приводит к более сильным повреждениям $n^{+}-p$-перехода.

Для исследуемых образцов в таблице представлены основные характеристики ФЭП, измеренные при условиях освещения АM1.5: напряжение холостого хода $U_{o c}$, плотность тока короткого замыкания $J_{s c}$, фактор заполнения $F F$, эффективность преобразования солнечного излучения $\eta$. Рост величин $U_{o c}, J_{s c}, F F$ и $\eta$ для образцов № 1-1, 1-2, 1-3, 2-1, 2-2, 3-1 по сравнению с аналогичными величинами для образца № 1 может быть объяснен как снижением отражательной способности, так и уменьшением $N_{t}$.

Образцы № 1-2, 2-2, 3-1 с наиболее высокими значениями $\eta$ характеризуются наибольшими величинами $J_{s c}$ в пределах подгруппы образцов с одинаковыми $C$ и наименьшими значениями $N_{t}$. Наблюдаемый рост $J_{s c}$ при снижении $N_{t}$ может объясняться уменьшением влияния процессов рекомбинации носителей с участием ГУ в области пространственного заряда $n^{+}-p$-перехода. Отмеченное снижение $N_{t}$ может быть объяснено удалением дефектов при травлении и эффектом пассивации при формировании por-Si [4-8]. Увеличение $t_{e t}$ в пределах подгруппы образцов с одинаковыми значениями $C$ (по сравнению с образцами № 1-2, 2-2, 3-1) приводит к росту $N_{t}$ и снижению величин $J_{s c}$ и $\eta$, что может объясняться ростом влияния рекомбинации с участием ГУ.

Наиболее высоким значением $\eta$ обладает образец № 2-2, содержащий только один ГУ $H 5$ с наименьшей концентрацией $\left(2.80 \cdot 10^{14} \mathrm{~cm}^{-3}\right)$. В то же время образец
ФЭП с наибольшей величиной $\eta$, в котором пленка por-Si формировалась анодным электрохимическим травлением, содержал ГУ $H 3$ и $H 5$ с концентрациями $2.31 \cdot 10^{14}$ и $2.67 \cdot 10^{15} \mathrm{~cm}^{-3}$ соответственно [4]. При этом образец № 2-2 и наиболее эффективный ФЭП, исследованный в [4], имеют близкие величины $\eta$ (14.10 и $14.27 \%$ соответственно). Это можно объяснить более высоким последовательным сопротивлением образца № 2-2 (3.4 $\Omega$ ) по сравнению с таковым для ФЭП из [4] $(2.9 \Omega)$. Оптимизация процесса формирования контактов к пленке por-Si с целью минимизации последовательного сопротивления ФЭП в данной работе не проводилась.

Таким образом, применение химического окрашивающего травления для формирования антиотражающей пленки por-Si на фронтальной поверхности ФЭП с $p$-n-переходом позволяет существенно снизить концентрацию дефектов с ГУ по сравнению с аналогичной величиной, полученной при использовании метода анодного электрохимического травления. Использование в качестве окислителя водного раствора $\mathrm{KMnO}_{4}$ при $C=0.025$ и $0.040 \mathrm{M}$ позволяет существенно увеличить время $t_{e t}$ (по сравнению с анодным электрохимическим травлением), при котором ФЭП имеют наиболее высокое значение $\eta$.

Представленные результаты получены в рамках выполнения государственного задания Министерства образования и науки РФ № 3.9506.2017/8.9 в Рязанском государственном университете им. С.А. Есенина. Спектры CDLTS измерялись на оборудовании Регионального центра зондовой микроскопии коллективного пользования при Рязанском государственном радиотехническом университете. 


\section{Список литературы}

[1] Handbook of porous silicon / Ed. L. Canham. Springer International Publ., 2014. $1017 \mathrm{p}$.

DOI: 10.1007/978-3-319-04508-5_95-1

[2] Ayat M., Belhousse S., Boarino L., Gabouze N., Boukherroub R., Kechouane M. // Nanoscale Res. Lett. 2014. V. 9. N 1. P. 482 (1-7). DOI: 10.1186/1556-276X-9-482

[3] Ermachikhin A.V., Litvinov V.G. // Instrum. Exp. Techn. 2018. V. 61. N 2. P. 277-282. DOI: $10.1134 / \mathrm{S} 0020441218020021$

[4] Трегулов В.В., Литвинов В.Г., Ермачихин А.В. // Письма в ЖТФ. 2017. Т. 43. В. 21. C. 3-9.

DOI: 10.21883/PJTF.2017.21.45155.16929

[5] Батенков В.А. Электрохимия полупроводников. Барнаул: Изд-во Алт. ун-та, 2002. $162 \mathrm{c.}$

[6] Улин В.П., Улин Н.В., Солдатенков Ф.Ю. // ФТП. 2017. T. 51. B. 4. C. 481-496.

DOI: 10.21883/PJTF.2019.04.47332.17597

[7] Стещюра С.В., Козловский А.В., Маляр И.В. // Письма в ЖТФ. 2015. Т. 41. В. 4. C. 24-32.

[8] Gautier G., Leduc P. // Appl. Phys. Rev. 2014. V. 1. N 1. P. 011101 (1-18). DOI: 10.1063/1.4833575 